\title{
AERODYNAMIC STUDIES IN THE STATIC COMPONENTS OF A CENTRIFUGAL COMPRESSOR STAGE
}

\author{
K. Srinivasa Reddy ${ }^{1}$, G.V. Ramana Murty ${ }^{2}$ and K.V. Sharma ${ }^{3}$ \\ ${ }^{1}$ Department of Mechanical Engineering, Aurora's Engineering College, \\ Bhongir, Nalgonda district, India; Phone: +918685-246309 \\ E-mail: kommareddy1970@gmail.com \\ ${ }^{2}$ Department of Mechanical Engineering, Vasavi College of Engineering, \\ Ibrahimbagh, Hyderabad, India; Phone: +9140-23146060 \\ ${ }^{3}$ Faculty of Mechanical Engineering, Universiti Malaysia Pahang, \\ 26600 Pekan, Pahang, Malaysia
}

\begin{abstract}
Aerodynamic studies in the static components of a centrifugal compressor stage were conducted using the computational fluid dynamics solver FLUENT. For the simulation study, a typical centrifugal compressor stage geometry with a flow coefficient of 0.053 was chosen, The study is confined to the static components of the centrifugal compressor stage, i.e., the crossover bend $\left(180^{\circ}\right.$ U-bend), a radial cascade of return channel vanes, and the exit ducting $\left(90^{\circ} \mathrm{L}\right.$-turn). The aerodynamic performance is reported in terms of total pressure loss coefficient, static pressure recovery coefficient, return channel vane surface static pressure distribution, and stage exit swirl angle distribution. The simulated flow through the static components covered five different operating conditions of the actual centrifugal compressor stage: the design point with $100 \%$ flow rate, and the off-design operating conditions with $70 \%, 80 \%, 110 \%$, and $120 \%$ flow rates. The standard k- $\varepsilon$ model was used with standard wall functions to predict the turbulence. A minimum total pressure loss coefficient was observed near $80 \%$ flow rate when the average flow angle at the U-bend inlet was $24^{\circ}$. Better static pressure recovery was observed with $70 \%, 80 \%$, and $100 \%$ flow rates. The swirl angle distribution at the stage exit was recognized as satisfactory.
\end{abstract}

Keywords: Return channel vanes; total pressure loss coefficient; static pressure recovery coefficient; vane surface static pressure coefficient; swirl angle.

\section{INTRODUCTION}

The development of modern centrifugal compressors demands extensive optimization of all the flow path components. In a multistage centrifugal compressor, the pressurized fluid from one stage is passed on to the subsequent stage via a $180^{\circ}$ circumferential Ubend, a cascade of de-swirl vanes known as "return channel vanes", and L-turn ducting with a $90^{\circ}$ bend for connection to the subsequent stage. The function of the return channel vanes is to guide the swirling flow leaving the diffuser to the eye of the subsequent impeller stage with near-zero inlet swirl. The flow through the U-bend and its interaction with the downstream row of de-swirl vanes presents a complex fluid dynamics problem. Aungier (2000, 1993) and Lüdtke (2004) presented some design guidelines for the design of return channel passages of a centrifugal compressor stage.

Reddy, Murty, Dasgupta and Sharma (2010) reported experimental and numerical studies in the crossover system of a typical centrifugal compressor stage with 
two different configurations of return channel vanes: RCV1, and RCV2. In their study, the ensemble of the static components of the centrifugal compressor stage, i.e., the crossover bend, cascade of return channel vanes, and the exit L-turn ducting, was referred to as the "crossover system". They conducted experimental investigations in a static model of 1:1 size simulating actual flow conditions under both design and offdesign operating conditions. The numerical studies were conducted by simulating the same flow conditions for the purpose of comparison. A good agreement between the experimental and numerical results was reported on the performance of the crossover system in terms of total pressure loss coefficient, static pressure recovery coefficient, and vane surface pressure distribution. The RCV1 configuration was observed to give better performance than RCV2. Simon \& Rothstein (1983) conducted experimental investigations on a static test rig with three different geometries of return channel vanes. They reported on the nature of flow through the return channel vanes and emphasized the need to describe the flow with the aid of simplified calculation models. In a similar fashion, Inoue and Koizumi (1983) conducted experimental investigations on a static test rig and reported the presence of secondary flow in the U-turn and the exit L-turn sections. They concluded that two-dimensional blades are adequate for the de-swirl vanes and that losses in the de-swirl vanes are due mainly to separation on the vane surfaces. Lenke and Simon (2000) conducted computational fluid dynamics (CFD) studies and showed that the deceleration of the flow introduces large separations and recirculation, which decreases the efficiency. They also demonstrated a qualitative agreement between the CFD results and the experimental data. An inverse design and optimization of a multistage radial compressor stage comprising a vaneless diffuser, crossover bend, and return channel, was presented by Veress and Braembussche (2004). They also studied the impact of vane lean on secondary flows and showed performance improvements with negative lean. Oh, Engeda and Chung (2005) solved the U-bend problem by using the FLUENT solver. It is shown that the wake/jet flow coming into the inlet of the U-turn bend develops secondary flow on the downstream side. An inverse method to design a circular cascade for the return channel of a centrifugal turbo machine, whose vane height varies in the radial direction, was developed by Toyokura, Kanemoto and Hatta (1986) using a singularity method. They also developed a circular cascade model and tested its performance experimentally. They concluded that the flow pattern through the cascade is very complex owing to interference between the secondary flow on the end wall and the flow separation on the vane surface.

In the present study, the aerodynamic performance of the static components, i.e., the U-bend, cascade of return channel vanes, and the exit L-turn ducting, is presented with a modified return channel vane configuration (RCV3). The aerodynamic performance of RCV3 is compared with that of RCV2 and RCV1 reported by Reddy et al. (2010). The numerical solution for the RCV3 configuration is obtained for the same operating conditions used in the study of RCV1 and RCV2. The computational details are described in the following section.

\section{COMPUTATIONAL DETAILS}

The numerical solution obtained by solving the Reynolds-Averaged Navier-Stokes (RANS) equations in relation to the present problem and solved by the code is given as follows. 
1. Conservation of mass

$$
\frac{\partial \rho}{\partial t}+\frac{\partial}{\partial x_{i}}\left(\rho u_{i}\right)=0
$$

2. Conservation of momentum

$$
\rho \frac{D u_{i}}{D t}=-\frac{\partial p}{\partial x_{i}}+\frac{\partial}{\partial x_{i}}\left[\mu\left(\frac{\partial u_{i}}{\partial x_{j}}+\frac{\partial u_{i}}{\partial x_{i}}-\frac{2}{3} \delta_{i j} \frac{\partial u_{k}}{\partial x_{k}}\right)\right]+\frac{\partial}{\partial x_{j}}\left(-\rho \overline{u_{i}^{\prime} u_{j}^{\prime}}\right)
$$

\section{Modeling Details}

The model is created using the GAMBIT preprocessor with three different blocks: crossover bend, return channel vane, and exit L-turn ducting. Three-dimensional sector models are used because the flow passages are axisymmetric. This procedure also minimizes the computer memory requirement and permits grid refinement in critical regions. The crossover bend and the L-turn ducting are meshed with structured hexahedral volumes. The return channel vane sector is meshed with unstructured hex/wedge volumes. Boundary layers are created on the vane surface to resolve the flow conditions at critical regions where flow separation is likely occur. The three blocks are coupled with the "interface" feature available in the program. The meridional view of the flow path is shown Figure 1. The meshed geometry of the three blocks is shown in Figure 2. Grid independence studies were carried out to determine the optimum grid size. The details of the grid independence studies are shown in Table 1. A grid generated with an interval spacing of $0.002 \mathrm{~m}$ is used in all simulations as this was established as the optimum grid interval size. The design details of the return channel vane configuration RCV3 are shown in Table 2. The flow path width at various locations is shown in Table 3.

Table 1. Grid independence studies.

\begin{tabular}{llll}
\hline Sl No. & $\begin{array}{l}\text { Grid interval size, } \\
(\mathrm{m})\end{array}$ & $\begin{array}{l}\text { Total number of } \\
\text { elements }\end{array}$ & $\begin{array}{l}\text { Total pressure } \\
\text { loss coefficient }\end{array}$ \\
\hline 1 & 0.003 & 72610 & 0.50185 \\
2 & 0.0025 & 131950 & 0.45027 \\
3 & 0.002 & 251460 & 0.4259 \\
4 & 0.0015 & 497232 & 0.4230 \\
\hline
\end{tabular}

Table 2. Flow path dimensions.

\begin{tabular}{lc}
\hline \multicolumn{1}{c}{ Location } & Flow path width $(\mathrm{mm})$ \\
\hline U-bend inlet & 24.5 \\
U-bend exit & 20.5 \\
L-turn exit & 47.5 \\
\hline
\end{tabular}




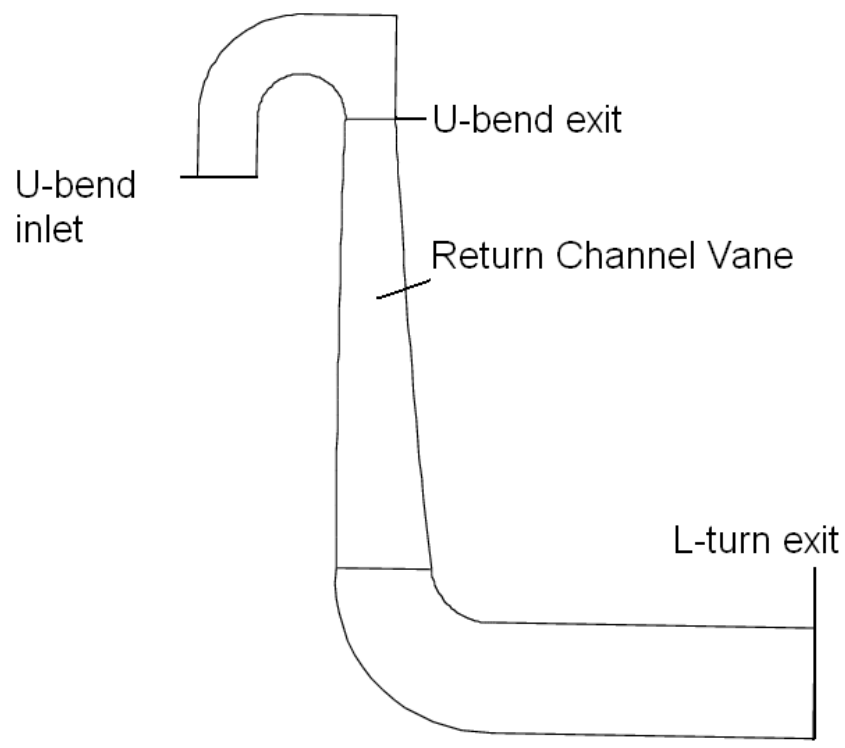

Figure 1. Meridional view of the flow path.
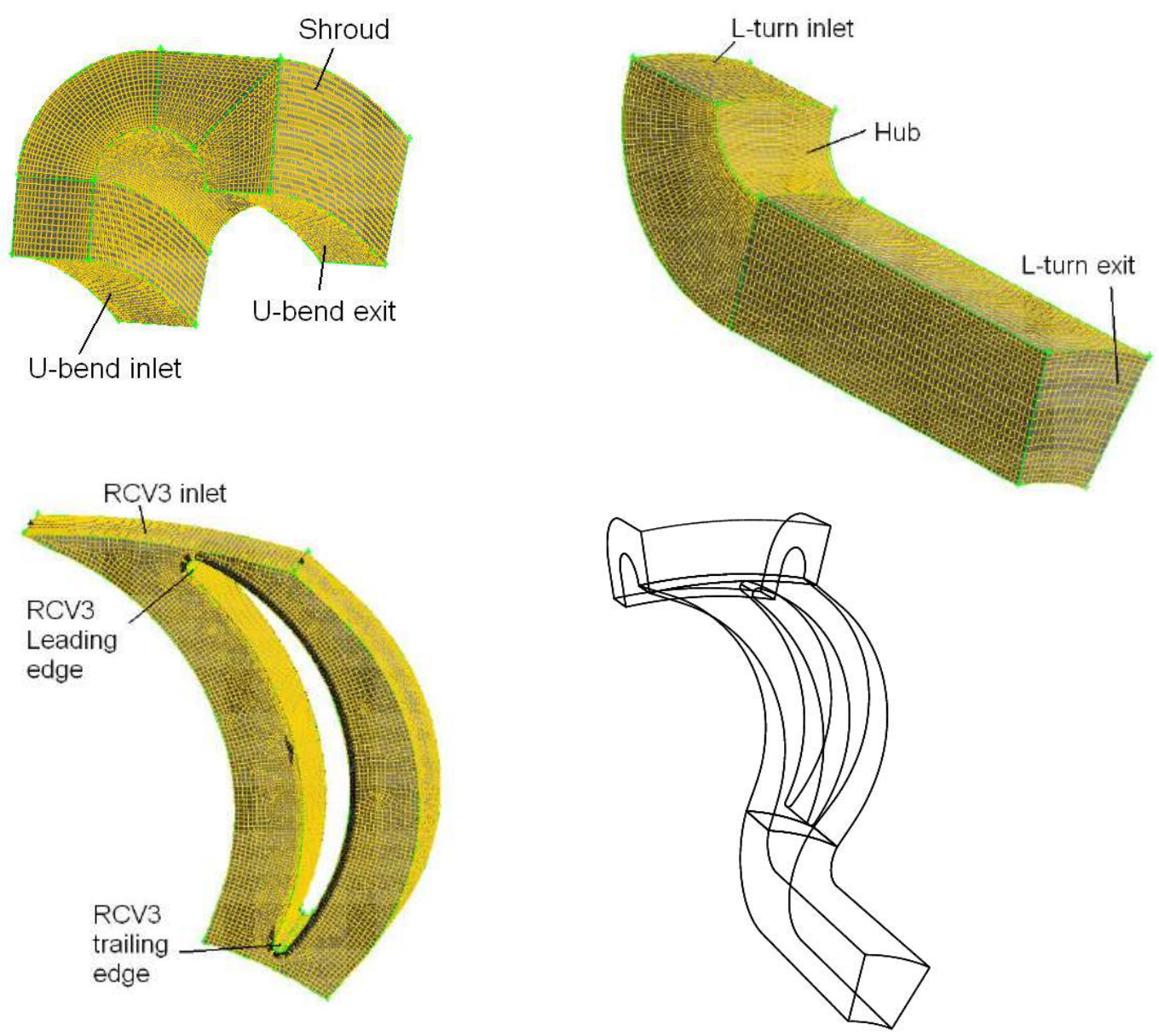

Figure 2. Meshed geometrical components and the combined model. 
Table 3. Vane parameters.

\begin{tabular}{ll}
\hline \multicolumn{1}{c}{ Parameter } & Value \\
\hline Inlet flow angle & $25^{\circ}$ \\
Exit flow angle & $82^{\circ}$ \\
Chord length & $178 \mathrm{~mm}$ \\
\hline
\end{tabular}

\section{Solver Settings}

A pressure-based solver with implicit formulation under 3-D steady flow conditions with absolute velocity formulation is chosen for the present study. Oh et al. (2005) conducted numerical studies of U-bends in return channel systems of multistage centrifugal compressors. They used a Reynolds stress turbulence model and a two equation $\mathrm{k}-\varepsilon$ turbulence model. In their study, the $\mathrm{k}-\varepsilon$ model predicted the turbulence closer to the experimental observations. In the present study, the $\mathrm{k}-\varepsilon$ model is used to predict the turbulence. Standard wall functions are used to capture the flow phenomenon near the walls. At the inlet section, "Total Pressure" is specified along with flow component directions. In addition, the density of air is specified. At the exit section of the $90^{\circ}$ bend, the "static pressure" with "radial equilibrium pressure distribution" option with target mass flow rate is used as the outlet boundary condition. The solution is assumed to converge when the maximum residual values are equal to $1 \times 10^{-6}$. The operating conditions (the average flow angle at the U-bend inlet and the corresponding mass flow rate) under which the simulations were carried out were taken based on the experimental investigations reported by Siva Reddy, Ramana Murty, Prasad and Reddy (2003).

\section{RESULTS AND DISCUSSION}

Figure 3(a) shows the variation of the meridional velocity from the hub to the shroud at the U-bend exit for the RCV3 configuration. Almost uniform velocities are observed from the hub to the shroud under all operating conditions. Higher magnitudes of meridional velocities are observed at this location than at the U-bend inlet under the given operating conditions. The increase in meridional velocity from the inlet to outlet of the U-bend may be attributed to the narrowing of the flow path width at the exit of the U-bend. The distribution of circumferentially averaged flow angle distribution from the hub to the shroud for the RCV3 configuration is shown in Figure 3(b). A uniform distribution in flow angle is observed under all the studied operating conditions. The magnitude of the average flow angle is seen to be higher at the U-bend exit than at the U-bend inlet under the given operating conditions. The increase in flow angle may also be attributed to the decreased flow path width at the U-bend exit. The decreased flow path width causes the meridional component to increase; hence, the increase in flow angle.

The vane surface pressure coefficient plotted against the percentage chord length from the leading edge is shown in Figure 4. The vane surface static pressure coefficient is obtained by dividing the static pressure difference between the local static pressure on the vane surface and the average static pressure at the U-bend inlet with average dynamic pressure at the U-bend inlet. The vane is observed loaded uniformly at the design point (average flow angle at U-bend inlet $=29^{\circ}$ ). With an increase in negative incidence on the leading edge of the RCV3 configuration, acceleration of the fluid is 
observed up to $30 \%$ chord length from the leading edge on the suction side and thereafter, deceleration of flow results in the recovery of static pressure towards the trailing edge. On the pressure side, immediately after the L-turn exit, a sudden drop in static pressure is noted at about 5\% chord length, which thereafter changes to positive static pressure values. This indicates possible flow separation at this zone and subsequent reattachment to the vane pressure surface, particularly at higher negative incidence angles.

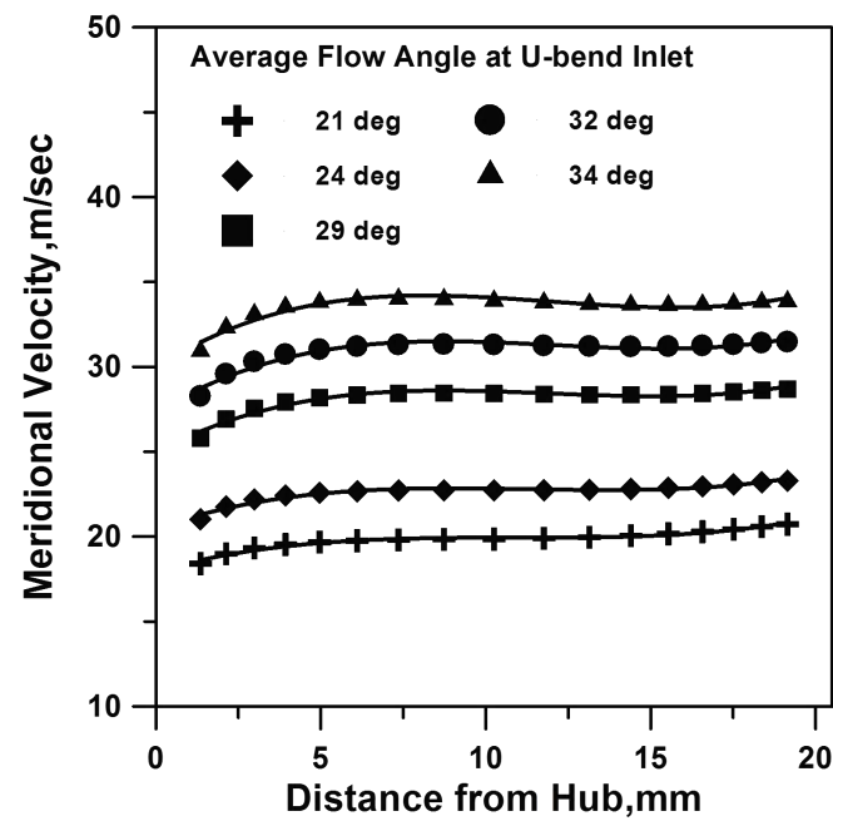

(a)

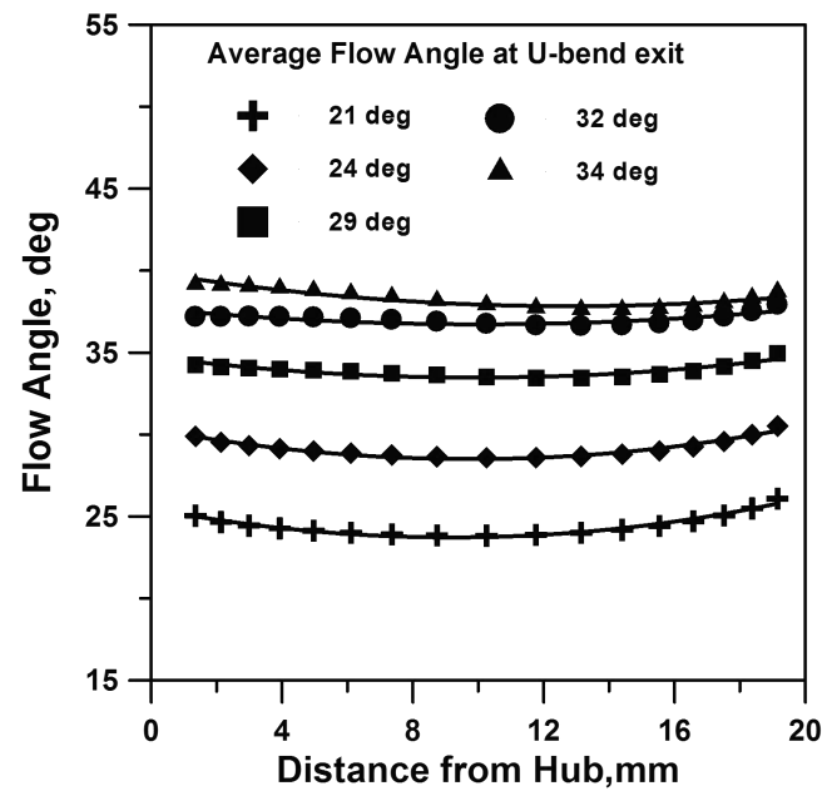

(b)

Figure 3 (a). Meridional velocity distribution; (b) Flow angle distribution at U-bend exit. 
The distribution of circumferentially averaged meridional velocity from the hub to the shroud at the L-turn exit is shown in Figure 5. The meridional velocity is concentrated more towards the hub and decreases in magnitude towards the shroud. Because of the increased mass flow rate caused by an increase in average flow angle at the U-bend inlet, increasing meridional velocities are observed. The L-turn causes the flow to be concentrated towards the hub.
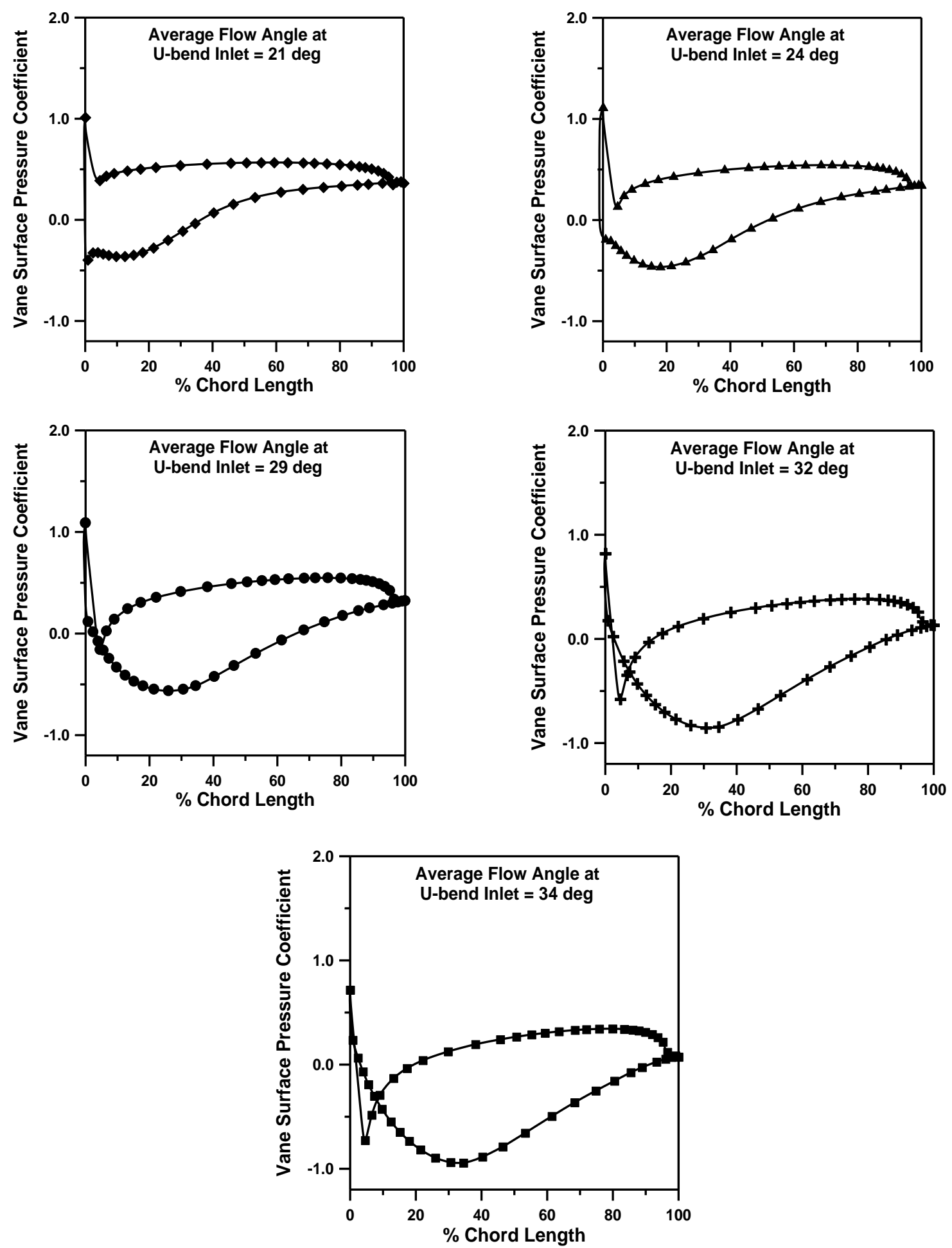

Figure 4. Vane surface static pressure distribution for RCV3 configuration. 


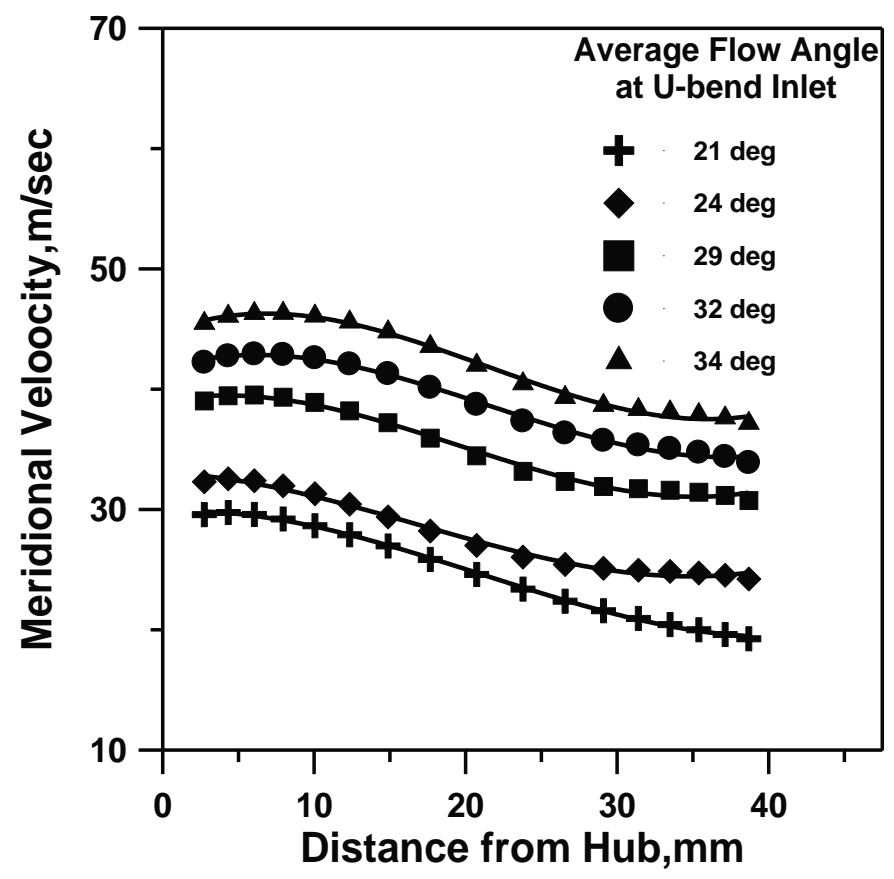

Figure 5. Meridional velocity distribution at L-turn exit.

Figure 6 shows the variation meridional velocity along the circumference at the L-turn exit section for the design point operating condition. A uniform meridional velocity distribution is observed near the hub, i.e., at a distance of $4.9 \mathrm{~mm}$ from the hub. With an increase in distance from the hub, the meridional velocity is seen decreasing in magnitude and varying in a wavy fashion. The wavy nature is observed to subside towards the hub. The L-turn bend appears to be causing the flow to migrate towards the hub. The vane trailing edge appears to have an effect on the meridional velocity distribution by causing the wavy nature of the variation.

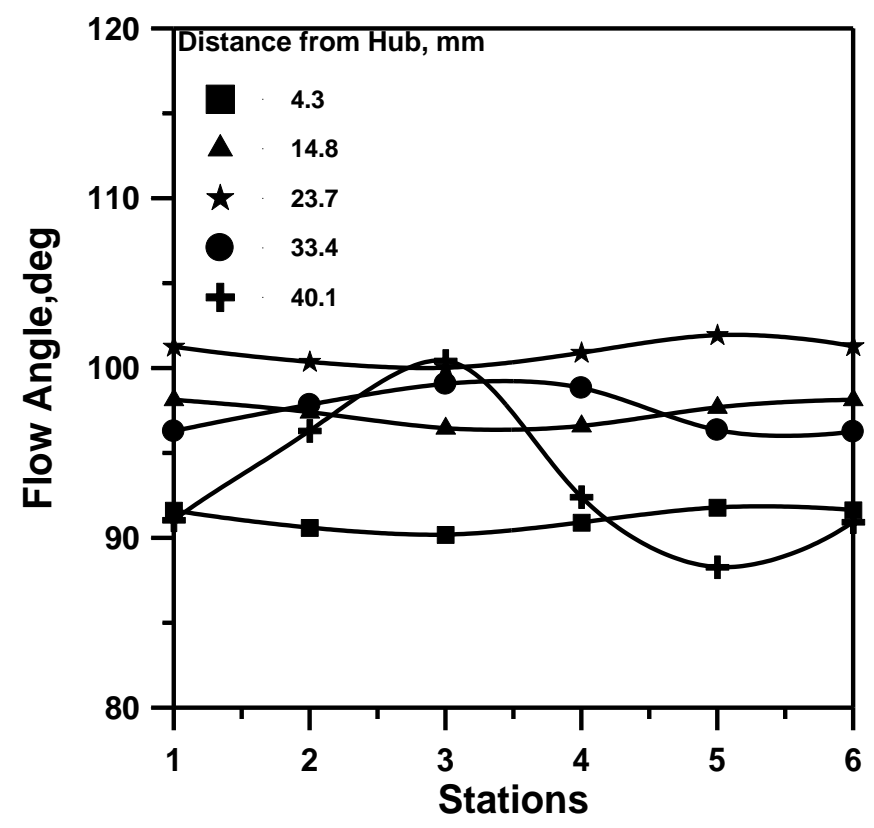

Figure 6. Meridional velocity distribution at L-turn exit (Design point). 
Figure 7 shows the variation of flow angle (swirl angle) at the L-turn exit section along the circumference at the design point. The flow angles are measured with respect to the tangential direction. Negative swirl (swirl angle greater than $90^{\circ}$ ) is observed in the mid-region of the L-turn section at distances of 14.8, 23.7, and $33.4 \mathrm{~mm}$ from the hub within the range of $96^{\circ}$ to $102^{\circ}$. Near the hub, a uniform and near-zero swirl angle distribution is observed. However, at a distance of $40.1 \mathrm{~mm}$ from the hub the swirl angle is observed to fluctuate between $88^{\circ}$ and $-101^{\circ}$ in a wavy fashion. The RCV3 vane trailing edge appears to have an effect on the nature of swirl angle distribution.

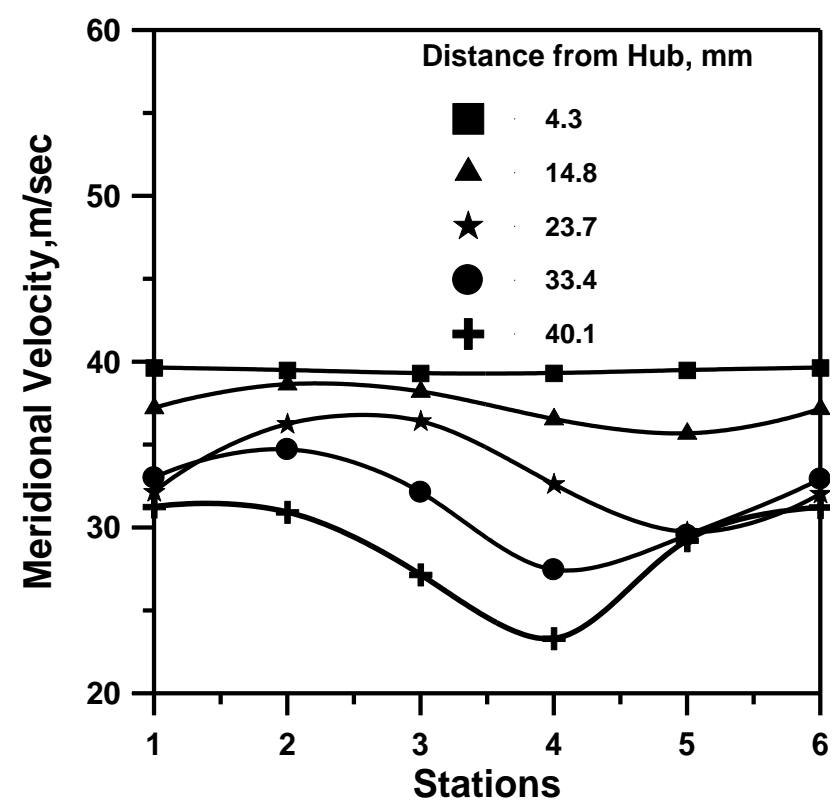

Figure 7. Swirl angle distribution at L-turn exit (Design point).

Figure 8 shows the variation of total pressure loss coefficient for RCV3, which is also compared with that of RCV1 and RCV2 reported by Reddy et al. (2010). The total pressure loss coefficient is obtained by dividing the average total pressure difference between the L-turn exit and the U-bend inlet with the average dynamic pressure at the U-bend inlet. The total pressure loss coefficient is observed to be a minimum for an average flow angle of $24^{\circ}$ at the U-bend inlet. The total pressure loss is seen to increase with an increase in flow angle at the U-bend inlet. The total pressure loss occurs owing to skin friction between the walls and the fluid and also because of internal friction arising due to secondary flows.

The variation of static pressure recovery coefficient with the average flow angles at the U-bend inlet is shown in Figure 9. The static pressure recovery coefficient is obtained by dividing the average static pressure difference between the L-turn exit and the U-bend inlet with the average dynamic pressure at the U-bend inlet. The static pressure is expected to recover well at lower incidence angles. An increase in incidence angle on the negative side, i.e., an increase in flow angle at the U-bend inlet, leads to a decrease in static pressure recovery. At higher negative incidence angles, the static pressure recovery coefficient is observed to be negative. The performance of the stage in terms of total pressure loss coefficient and the static pressure recovery for RCV3 is observed to be superior to RCV1 and RCV2. 


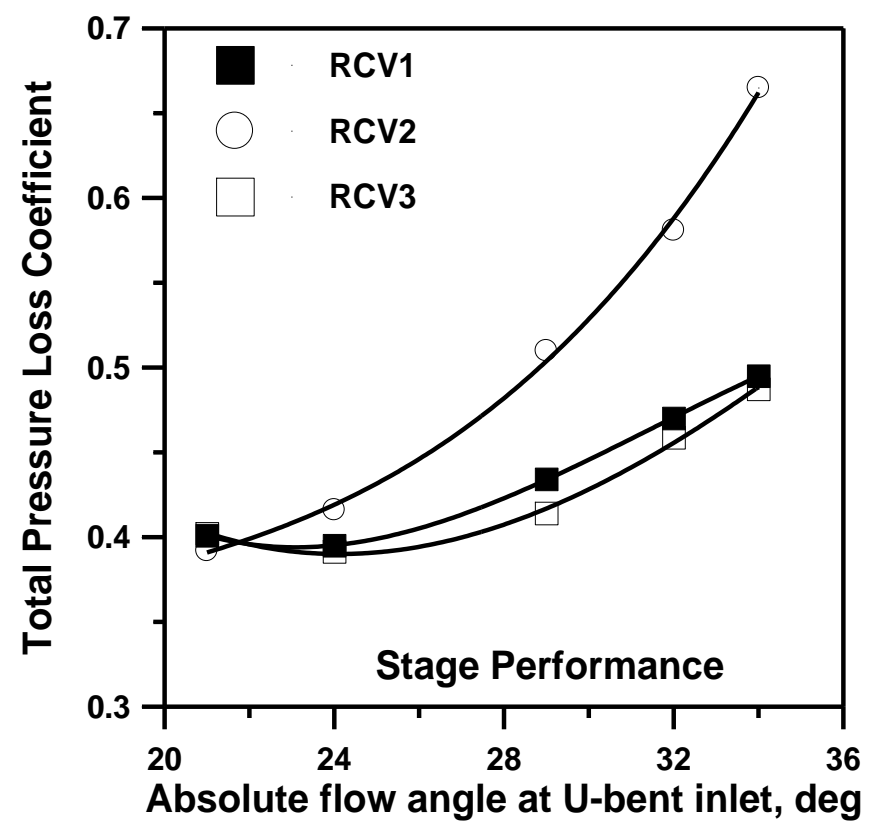

Figure 8. Variation of total pressure loss coefficient.

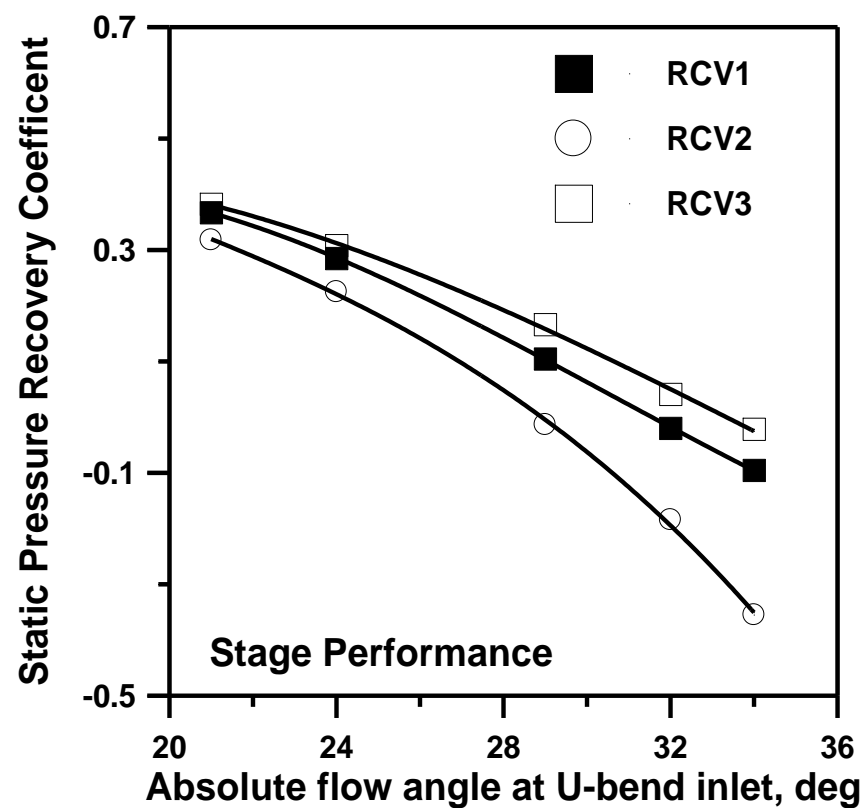

Figure 9. Variation of static pressure recovery coefficient.

\section{Qualitative Plots}

The flow separation phenomenon observed occurring on the suction side of the return channel vane trailing edge in the studies of RCV1 and RCV2, is almost eliminated in the case of RCV3. Figure 10 shows the qualitative velocity vector plots depicting the flow on the suction side of the trailing edge at the design point. Figure 10(a) shows the velocity vector plots on a plane passing the mid-span of the vane for the three vane configurations. Figure 10(b) shows the magnified views of the flow on the suction side 
of the trailing edge. The flow separation phenomenon is observed eliminated in the case of the RCV3 configuration, as can be seen in the magnified vector plots.
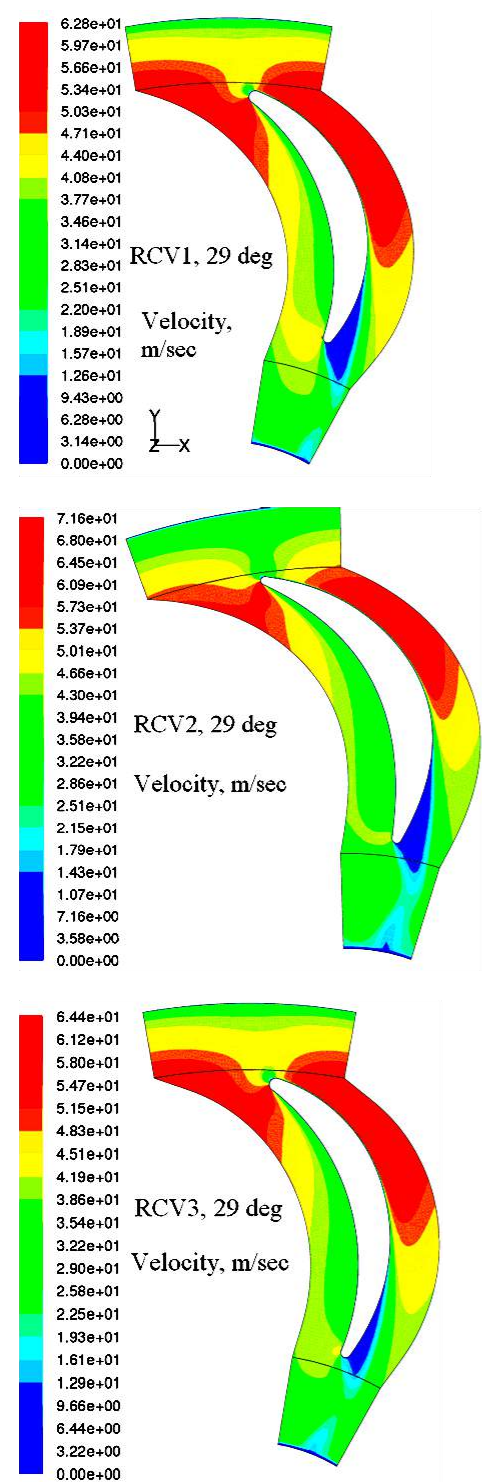

(a)
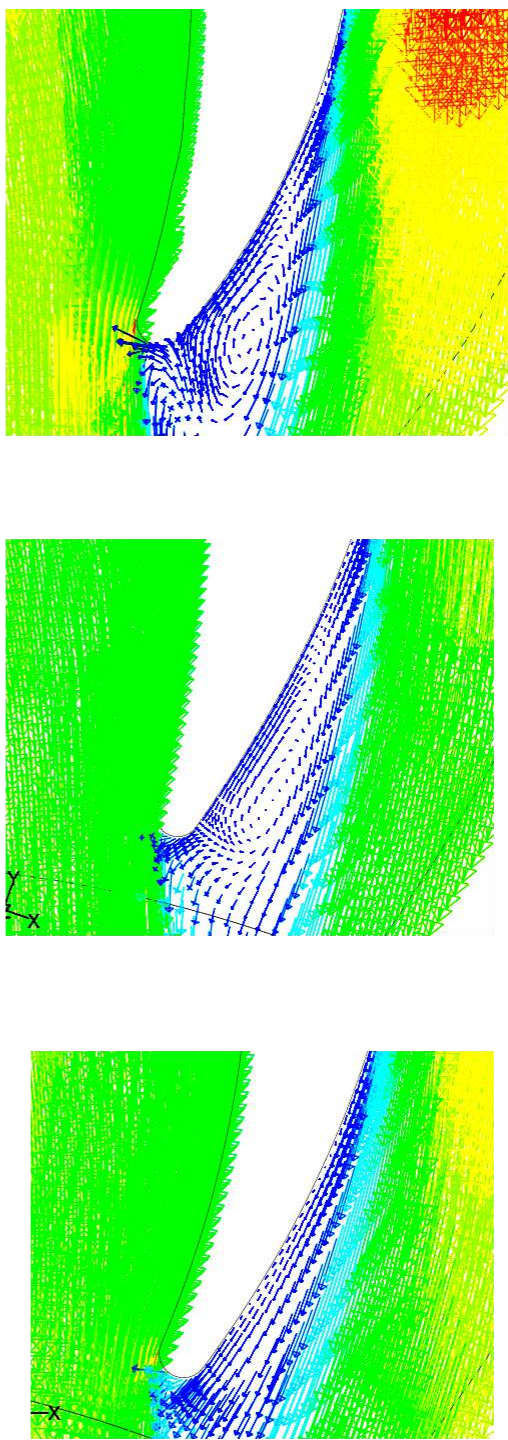

(b)

Figure 10. Velocity vector plots on a plane passing through the mid-span of the vane.

\section{CONCLUSIONS}

A numerical study was conducted to study the aerodynamic performance of the static components of a centrifugal compressor stage with a specific vane configuration, namely RCV3. Uniform velocity and flow angle distributions were observed at the Ubend exit. The flow separation phenomenon that was observed in the RCV1 and RCV2 configurations is eliminated with the RCV3 configuration. The meridional velocity and swirl angle distributions are observed to be influenced by the return channel vane trailing edge. The stage exit swirl angle distribution is observed to vary between $80^{\circ}$ and $102^{\circ}$. The present study is found helpful in understanding the nature of flow through the return channel passages of a centrifugal compressor stage. 


\section{REFERENCES}

Aungier, R. H. (1993). Aerodynamic design and analysis of vaneless diffusers and return channels. ASME, Paper No. 93-GT-101.

Aungier, R. H. (2000). Centrifugal compressors. New York: ASME Press.

Inoue, Y., \& Koizumi, T. (1983). Experimental study on flow patterns and losses in return passages for centrifugal compressor. ASME Fluids engineering division, 3, 13-21.

Lenke, L. J., \& Simon, H. (2000). Aerodynamic analysis of return channels of multistage centrifugal compressors. ASME PID-Vol. 5, Challenges and Goals in Industrial and Pipeline Compressors.

Lüdtke, K. H. (2004). Process centrifugal compressors. Berlin: Springer.

Oh, J. M., Engeda, A., \& Chung, M. K. (2005). A numerical study of the U-turn bend in return channel systems for multistage centrifugal compressors. Proceedings of the Institution of Mechanical Engineers, Part C: Journal of Mechanical Engineering Science, 219(8), 749-756.

Reddy K. S., Murty, G. V. R., Dasgupta, A., \& Sharma, K. V. (2010). Flow investigations in the crossover system of a centrifugal compressor stage. International Journal of Fluid Machinery and Systems, 3(1), 11-19.

Simon, H., \& Rothstein, E. (1983). On the development of return passages of multistage centrifugal compressors. ASME Fluids Engineering Division, Return Passages of Multistage Turbo machinery, 3, 1-11.

Siva Reddy, T. C., Ramana Murty, G. V., Prasad, M. V. S. S. S. M., \& Reddy, D. N. (2003). Theoretical and experimental studies on low solidity Vaned diffusers of a centrifugal compressor stage. Proceedings of 30th National Conference on Fluid Mechanics and Fluid Power, December 11-13, NIT, Surathkal, India, pp. 621628.

Toyokura, T., Kanemoto, T., \& Hatta, M. (1986). Studies on circular cascades for return channels of centrifugal turbomachinery. Bulletin of JSME, 29(255), 2885-2892.

Veress, A., \& Braembussche, R. V. (2004). Inverse design and optimization of a return channel for a multistage centrifugal compressor. ASME, Journal of Fluids Engineering, 126(5), 799-806. 